\title{
Unusual Dielectric Loss Properties of Carbon Nanotube-Polyvinylidene Fluoride Composites in Low Frequency Region (100 Hz $<$ f $<1$ MHz)
}

\author{
Yi Zhen, Juan Arredondo, Guang-Lin Zhao \\ Department of Physics, Southern University and A\&M College, Baton Rouge, USA \\ Email: yi_zhen@subr.edu, Guang-Lin_Zhao@subr.edu
}

Received August 13, 2013; revised September 13, 2013; accepted September 20, 2013

Copyright (C) 2013 Yi Zhen et al. This is an open access article distributed under the Creative Commons Attribution License, which permits unrestricted use, distribution, and reproduction in any medium, provided the original work is properly cited.

\begin{abstract}
Systematic investigations on the dielectric properties of multi-walled carbon nanotubes (MWNTs)-polyvinylidene fluoride (PVDF) composites with a wide MWNT concentration range (2 - $9 \mathrm{wt} \%)$ have been carried out. It is revealed that the dielectric constant is increased by the addition of an appropriate amount of MWNTs at room temperature. However, when the concentration of MWNTs in the composites reaches above $5 \mathrm{wt} \%$, negative dielectric constants and large dielectric loss in the composites are observed in the low frequency range. The ferroelectric CNT-PVDF polymer composites containing more than $5 \mathrm{wt} \%$ MWNTs have a strong dielectric absorption, which has the potential for acoustic applications.
\end{abstract}

Keywords: Polymer Composites; Carbon Nanotube; Dielectric Properties

\section{Introduction}

The inclusion of nanoscale subjects in a matrix material such as polymers can lead to substantial enhancement in selected physical properties relative to the pristine material. The significant improvements can be achieved with a relatively low loading of the nanofillers due to the high surface area to volume ratio. Carbon nanotube (CNT) has attracted intensive interest due to their nanometer-scale dimension as well as their large shape anisotropy and unique mechanical, thermal, and electrical properties [1, 2]. Because of their large dielectric constant, electrically conductive carbon nanotubes are attractive as fillers for high dielectric-constant nanocomposites. In this work, we are particularly interested in the dielectric loss properties of carbon nanotubes - polyvinylidene fluoride (PVDF) composites. PVDF is a highly non-reactive and pure thermoplastic fluoropolymer. Below $150^{\circ} \mathrm{C}$, PVDF becomes ferroelectric [3,4]. Thus, PVDF is electroactive and semicrystalline polymer with pyro and piezoelectric properties at room temperature, which have led to many technological applications. Its acoustic impedance similar to that of water or living tissue makes it very desirable for acoustic applications. Its high permittivity and relatively low dissipation factor made it to be chosen as host polymers to perform efficient electrical-to-mechanical energy conversion and mechanical actuation under external electrical stimulation $[5,6]$. The dielectric properties of CNT-PVDF composites can be tailored to suit particular performance requirements for some special applications [7-10]. The composites are many-phase systems and their properties are affected essentially by parameters such as the preparation method of the composites and the dispersion of CNTs into the polymer matrix [11-15].

In this paper, we report the observed unusual dielectric properties of multi-walled carbon nanotubes (MWNTs) - PVDF composites in a frequency range from $100 \mathrm{~Hz}$ to $1 \mathrm{MHz}$. The results include the observations of negative dielectric constants and large dielectric loss in the composites, especially in low frequency region.

\section{Experimental Section}

\subsection{Materials}

The semicrystalline polymer PVDF powders were purchased from a supplier company Alfa Aesar for reagents and materials (\#CAS 24937-79-9, melting point: $155^{\circ} \mathrm{C}$ $160^{\circ} \mathrm{C}$ ). Multi-walled carbon nanotubes (MWNTs) were obtained from a commercial producer (Cheaptubes, USA). The MWNTs have the outer diameters (OD) less than $8 \mathrm{~nm}$. The purity of MWNTs is better than $95 \%$. 
The ash content in the MWNTs powder is less than 1.5 $\mathrm{wt} \%$. The length distribution of MWNTs is in the range of $10-30 \mu \mathrm{m}$. The MWNTs specific surface area is about $500 \mathrm{~m}^{2} / \mathrm{g}$. The DC electrical conductivity of the MWNTs is higher than $100 \mathrm{~S} / \mathrm{cm}$. MWNTs have much higher performance-to-price ratio (PPR) than that of single-wallcarbon nanotubes (SWNTs) for large scale composite applications.

\subsection{Sample Preparation}

The PVDF powders were firstly dissolved in an organic solvent(1-Methyl-2-Pyrrolidinone). MWNTs were then added into PVDF solution according to the weight percentages of MWNTs $(2 \%-9 \% \mathrm{wt} / \mathrm{wt})$; and the mixture was stirred for 1 hour to achieve a uniform dispersion. After that, the solution was poured on glass substrate and then underwent a thermal treatment at $120^{\circ} \mathrm{C}$ for $2 \mathrm{~h}$.

\subsection{Measurements}

For the measurements of dielectric properties of the MWNT-PVDF samples, silver paste was applied to the surface of composite samples as the electrodes. The dielectric constant and loss were measured by using a HP Model 4284A LCR meter in a frequency range from 100 $\mathrm{Hz}$ to $1 \mathrm{MHz}$ in a constant temperature furnace.

\section{Results and Discussion}

\subsection{Characterization of MWNT-PVDF Composites}

Figure 1 shows the scanning electron microscopy (SEM) micrographs for the fractured surface morphologies of MWNT-PVDF composite samples. It is known that MWNTs can spontaneously attract each other to form MWNTs bundles, due to weak van der Waals forces between them. As the loading of MWNTs increased to 5 $w \mathrm{t} \%$, MWNTs bundles form aggregates in the composite as shown in Figure 1(b). Figure 1(c) shows the network of MWNT aggregates and agglomerates with irregular sizes and shapes in the PVDF matrix when the loading of MWNT reaches $9 \mathrm{wt} \%$.

\subsection{Dielectric Properties of MWNT-PVDF Composites}

Figure 2 shows the frequency dependences of the dielectric constant $\left(\varepsilon^{\prime}\right)$ of MWNT-PVDF composites at $40^{\circ} \mathrm{C}$ for the MWNT loadings from $2 \%$ to $9 \% \mathrm{wt} / \mathrm{wt}$. The dielectric constant of MWNT-PVDF composites is higher than that of the PVDF $\left(\varepsilon^{\prime} \sim 10\right)$. With high MWNT concentrations in the composite, the values of the dielectric constant and its frequency dependence are quite much different from those with low MWNT loadings. The di-

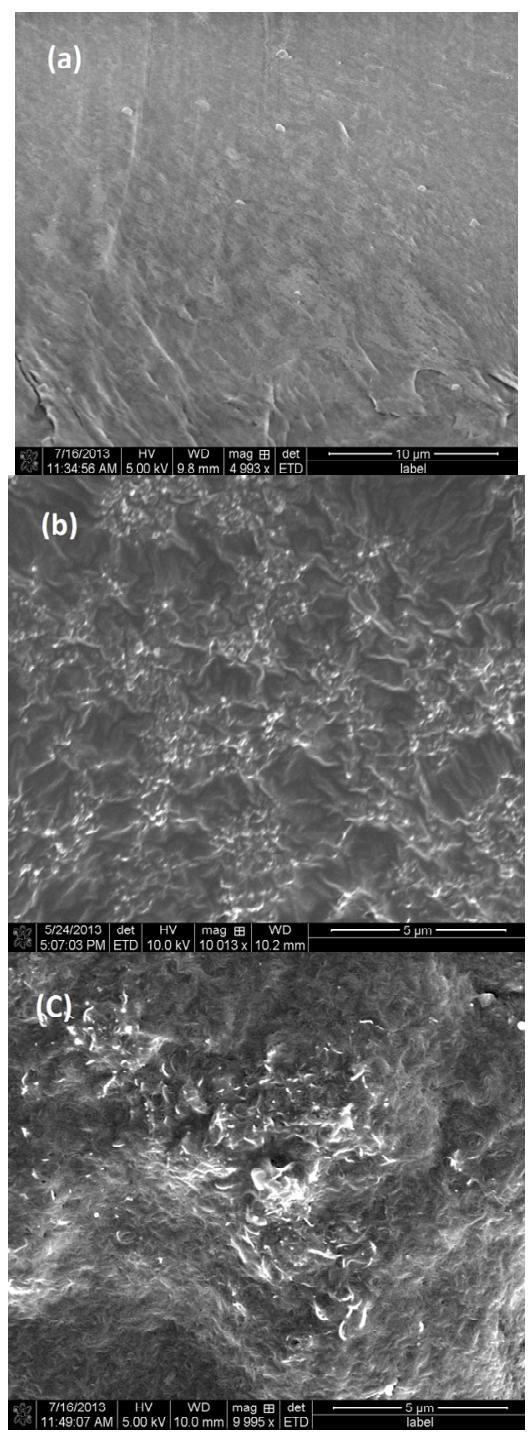

Figure 1. SEM micrographs of MWNT-PVDF composites with the MWNT loadings of 0 wt \% (a), 5 wt\% (b), and 9 wt\% (c).

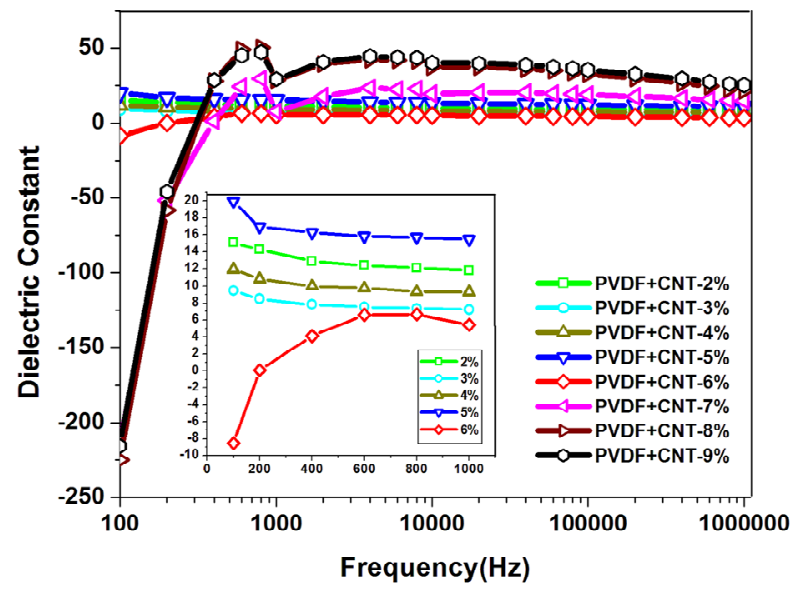

Figure 2. Frequency-dependence of the dielectric constant of MWNT-PVDF at $40^{\circ} \mathrm{C}$. 
electric constants of the composites with MWNT loading less than $5 \mathrm{wt} \%$ show a weak frequency dependence. However, when the MWNT loading is higher than $6 \mathrm{wt} \%$, the dielectric constants of the composite samples show strong frequency dependence in the frequency range from $10^{2} \mathrm{~Hz}$ to $10^{6} \mathrm{~Hz}$. The feature is especially very pronounced in the low frequency region (lower than 10 $\mathrm{KHz}$ ). The dielectric constants of the MWNT-PVDF composites can become negative at low frequency when the MWNT loadings in the composite samples beyond 6 wt $\%$. At $100 \mathrm{~Hz}$, the dielectric constants become about -225 for the composite samples with $7-9 \mathrm{wt} \%$ MWNTs. As the frequency increases, the dielectric constants of the composites increase and then become positive when the frequencies are higher than $300 \mathrm{~Hz}$. In higher frequency range from $10^{4} \mathrm{~Hz}$ to $10^{6} \mathrm{~Hz}$, the dielectric constants of the composites are positive at about 30 , and show a weak frequency dependence.

In the measurements, the LCR meter firstly obtained the admittance $Y=\frac{I}{V}=\frac{R-i\left(X_{L}-X_{C}\right)}{R^{2}+\left(X_{L}-X_{C}\right)^{2}}$, through voltage $\mathrm{V}$ and current $\mathrm{I}$, where $\mathrm{R}$ is resistance, $X_{C}=\frac{1}{2 \pi f C}$ is capacitive reactance, and $X_{L}=2 \pi f L$ is inductive reactance. If $X_{L}>X_{C}$, the admittance will have a negative imaginary part, which leads to a switch of the sign of the phase difference between current and voltage. The calculation of the VEE data analysis/software of the HP LCR meter will show a negative dielectric constant. Physically, the negative dielectric constant from the measurements is due to a change of the material property from more capacitive to more inductive behavior. Increasing the MWNT loading higher than $6 \mathrm{wt} \%$, the MWNTPVDF composite will become more conductive and also have an inductive behavior, due to the nature of MWNT wires in the composite material.

Figure 3 shows the frequency dependence of dielectric loss, which is also the imaginary part of the complex permittivity, of the MWNT-PVDF composites at $40^{\circ} \mathrm{C}$. The dielectric loss is substantially increased by increasing the MWNT weight percentages in the composites above $5 \mathrm{wt} \%$. The feature is especially very pronounced in the low frequency region (lower than $10 \mathrm{KHz}$ ). The dielectric loss becomes several hundred in the low frequency region, when the composite materials contain more than $5 \mathrm{wt} \%$ MWNTs. The largest value of the dielectric loss is 1870 at $200 \mathrm{~Hz}$ for the composite material containing $9 \mathrm{wt} \%$ MWNTs. These results show that the ferroelectric polymer composites containing more than 5 wt $\%$ MWNTs have a strong dielectric absorption, which has the potential for acoustic applications via pyro and piezoelectric effect. The increased dielectric loss is related to the increase in electrical conductivity due to the

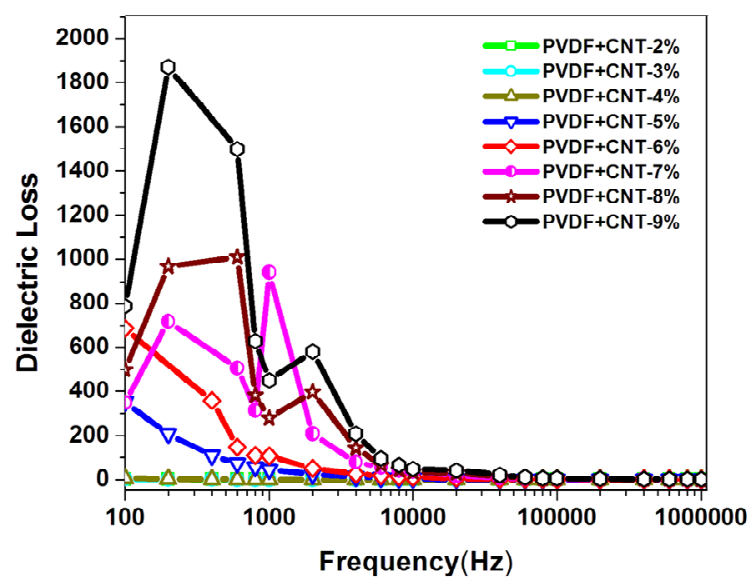

Figure 3. Frequency-dependence of the dielectric loss of MWNT-PVDF composites at $40^{\circ} \mathrm{C}$.

effect of the electrically conductive MWNTs. The strong dielectric loss of the composites indicates that the addition of MWNTs increases the concentration of the mobile charges in the composites significantly when the MWNT concentration is above $5 \mathrm{wt} \%$. When the frequency is higher than $10 \mathrm{KHz}$, the dielectric loss decreases to 2.73 and show weak frequency dependence.

In order to take a closer look at the dielectric loss, the dielectric modulus is used. An dielectric modulus $\mathrm{M}$ is defined by $M=\left(\varepsilon^{*}\right)^{-1}$, where $\varepsilon^{*}=\varepsilon^{\prime}-i \varepsilon^{\prime \prime}$ is the complex dielectric permittivity; $\varepsilon^{\prime}$ and $\varepsilon^{\prime \prime}$ are the real and imaginary part of dielectric permittivity. The dielectric modulus $M$ was originally introduced to study space charge relaxation phenomena and is now widely used to analyze ionic conductivities. The dielectric modulus corresponds to the relaxation of the electric field in the material when the electric displacement remains constant. The imaginary part of dielectric modulus is given by $\left.M^{\prime \prime}=\varepsilon^{\prime \prime}\right]\left(\varepsilon^{\prime 2}+\varepsilon^{\prime \prime 2}\right)$. Figure 4 shows the imaginary part of the dielectric modulus M for pure PVDF and MWNTPVDF composites in the frequency range from $100 \mathrm{~Hz}$ to $1 \mathrm{MHz}$ at various temperature. It is noticed that when the MWNT loading is above $5 \mathrm{wt} \%$ in the composites, a peak structure is observed in dielectric modulus spectra. The peak shifts to higher frequency when the concentration of MWNTs in the composites is increased. $M^{\prime \prime}$ increases as the temperature increases on the low frequency side of peak. The low frequency side of the peak represents the range of frequencies in which the charges are able to move a relatively long distance. Whereas, for the high frequency side, the charges are not able to response to alternating electric field and they can only execute localized motion [16].

\section{Conclusion}

In conclusion, the composites of MWNT-PVDF with 2 - 


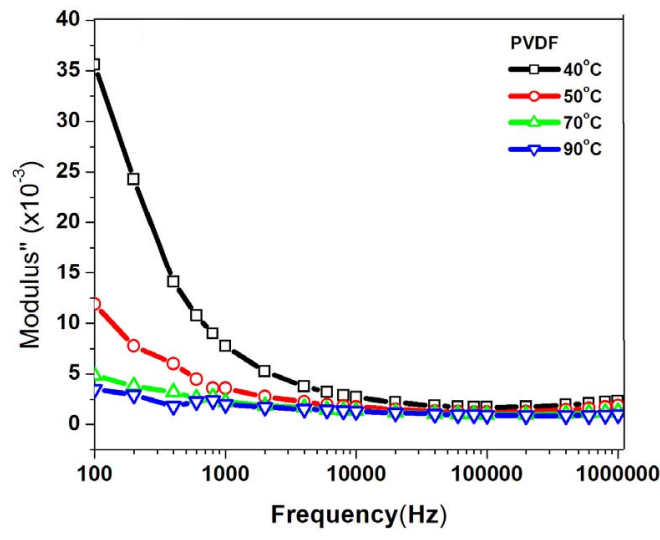

(a)

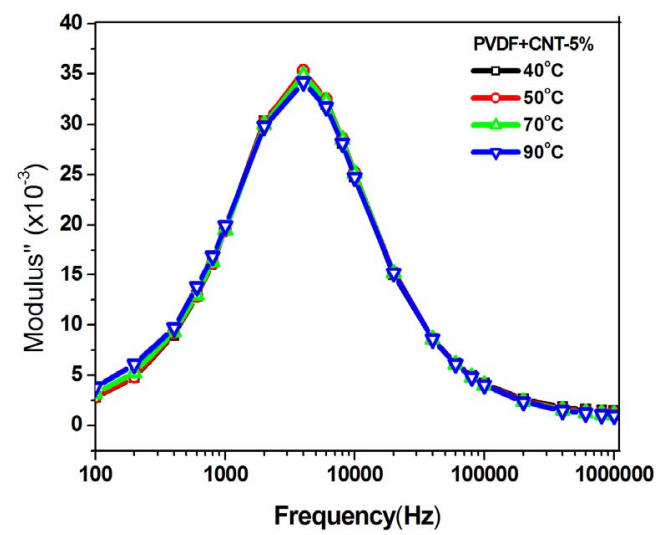

(b)

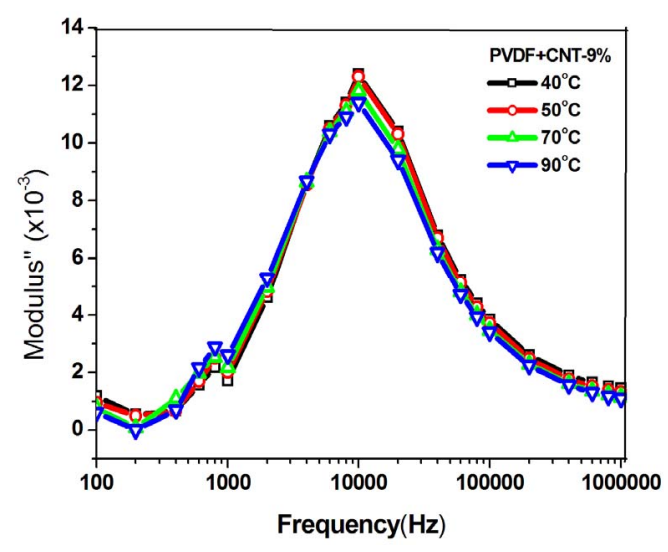

(c)

Figure 4. Dielectric modulus spectra of PVDF and MWNTPVDF composites with 0 wt \% (a), 5 wt \% (b) and 9 wt\% (c) at various temperatures.

$9 \mathrm{wt} \%$ MWNT loadings have been synthesized. The dielectric properties of the MWNT-PVDF composites in the frequency range from $100 \mathrm{~Hz}$ to $1 \mathrm{MHz}$ have been studied. The dielectric constant and loss properties of MWNT-PVDF composites show an important change at the threshold of $5 \mathrm{wt} \%$ MWNT loading. When the MWNT concentration in the composite is larger than 5 $\mathrm{wt} \%$, the magnitude of the dielectric constant of the com- posite increase significantly and the dielectric constant appears to be negative in the frequency range between $100 \mathrm{~Hz}$ and $1000 \mathrm{~Hz}$, while dielectric loss increases substantially. The ferroelectric CNT-PVDF polymer composites containing more than $5 \mathrm{wt} \%$ MWNTs have a strong dielectric absorption, which has the potential for acoustic applications via pyro and piezoelectric effect.

\section{Acknowledgements}

The work was funded in part by the Air Force Office of Scientific Research (Award No FA9550-09-1-0367 and FA9550-11-1-0330), the National Science Foundation (Award No CBET-0754821), and NSF LASIGMA Project (Award No. EPS-1003897, NSF92010-15-RIISUBR).

\section{REFERENCES}

[1] Q. M. Zhang, H. F. Li, M. Poh, F. Xia, Z. Y. Cheng, H. S. $\mathrm{Xu}$ and C. Huang, "An All-Organic Composite Actuator Material with a High Dielectric Constant," Nature, Vol. 419, 2002, pp. 284-287. http://dx.doi.org/10.1038/nature01021

[2] C. Huang, R. Klein, F. Xia, H. Li, Q. M. Zhang, F. Bauer and Z. Y. Cheng, "Poly(vinylidene Fluoride-Trifluoroethylene) Based High Performance Electroactive Polymers," IEEE Transactions on Dielectrics and Electrical Insulation, Vol. 11, No. 2, 2004, pp. 299-311. http://dx.doi.org/10.1109/TDEI.2004.1285901

[3] H. J. Kawai, "The Piezoelectricity of Poly(vinylidene Fluoride), "The Piezoelectricity of Poly (vinylidene Fluoride)," Japanese Journal of Applied Physics, Vol. 8, 1969, pp. 975-976. http://dx.doi.org/10.1143/JJAP.8.975

[4] M. G. Broadhurst and G. T. Davis, "Physical Basis for Piezoelectricity in PVDF," Ferroelectrics, Vol. 60, No. 1, 1984, pp. 3-13.

http://dx.doi.org/10.1080/00150198408017504

[5] S. Ahmed and F. R. Jones, "A Review of Particulate Reinforcement Theories for Polymer Composites," Journal of Materials Science, Vol. 25, No. 12, 1990, pp. 49334942. http://dx.doi.org/10.1007/BF00580110

[6] R. Jr. Gregorio and M. Cestari, "Effect of Crystallization Temperature on the Crystalline Phase Content and Morphology of Poly(vinylidene Fluoride)," Journal of Polymer Science, Part B: Polymer Physics, Vol. 32, No. 5, 1994, pp. 859-870. http://dx.doi.org/10.1002/polb.1994.090320509

[7] L. Wang and Z-.M. Dang, "Carbon Nanotube Composites with High Dielectric Constant at Low Percolation Shreshold," Applied Physics Letters, Vol. 87, No. 4, 2005, Article ID: 042903. http://dx.doi.org/10.1063/1.1996842

[8] J. K. Yuan, W. L. Li, S. H. Yao, Y. Q. Lin, A. Sylvestre and J. B. Bai, "High Dielectric Permittivity and Low Percolation Threshold in Polymer Composites Based on SiCCarbon Nanotubes Micro/Nano Hybrid," Applied Physics Letters, Vol. 98, No. 3, 2011, Article ID: 032901. http://dx.doi.org/10.1063/1.3544942

[9] S. H. Zhang, N. Y. Zhang, H. Cheng, K. L. Ren and Q. M. 
Zhang, "Microstructure and Electromechanical Properties of Carbon Nanotube/Poly(vinylidene Fluoride-Trifluoroethylene-Chlorofluoroethylene) Composites," Advanced Materials, Vol. 17, No. 15, 2005, pp. 1897-1901. http://dx.doi.org/10.1002/adma.200500313

[10] J. K. Yuan, S. H. Yao, Z. M. Dang, A. Sylvestre, M. Genestoux and J. B. Bai, "Giant Dielectric Permittivity Nanocoposites: Realizing True Potential of Pristine Carbon Nanotubes in Polyvinylidene Fluoride Matrix through an Enhanced Interfacial Interaction," The Journal of Physical Chemistry C, Vol. 115, No. 13, 2011, pp. 5515-5521. http://dx.doi.org/10.1021/jp1117163

[11] C. K. Chiang and R. Popielarz, "Polymer Composites with High Dielectric Constant," Ferroelectrics, Vol. 275, No. 1, 2002, pp. 1-9. http://dx.doi.org/10.1080/00150190214285

[12] G. C. Psarras, K. G. Gatos, P. K. Karahaliou, S. N. Georga, C. A. Krontiras and J. Karger-Kocsis, "Relaxation Phenomena in Rubber/Layered Silicate Nanocomposites," eXPRESS Polymer Letters, Vol. 1, 2007, pp. 837-845. http://dx.doi.org/10.3144/expresspolymlett.2007.116
[13] H. Ishida, S. Campbell and J. Blackwell, "General Approach to Nanocomposite Preparation," Chemistry of Materials, Vol. 12, No. 5, 2000, pp. 1260-1267. http://dx.doi.org/10.1021/cm990479y

[14] Q. Li, Q. Z. Xue, X. L. Gao and Q. B. Zheng, "Temperature Dependence of the Electrical Properties of the Carbon Nanotube/Polymer Composites," eXPRESS Polymer Letters, Vol. 3, 2009, pp. 769-777. http://dx.doi.org/10.3144/expresspolymlett.2009.95

[15] C. V. Chanmal and J. P. Jog, "Dielectric Relaxations in PVDF/BaTiO ${ }_{3}$ Nanocomposites," eXPRESS Polymer Letters, Vol. 2, 2008, pp. 294-301. http://dx.doi.org/10.3144/expresspolymlett.2008.35

[16] J. J. Liu, C. G. Duan, W. G. Yin, W. N. Mei, R. W. Smith and J. R. Hardy, "Dielectric Permittivity and Electric Modulus in $\mathrm{Bi}_{2} \mathrm{Ti}_{4} \mathrm{O}_{11}$," Journal of Chemical Physics, Vol. 119, 2003, pp. 2812-2819. http://dx.doi.org/10.1063/1.1587685 